\title{
Study of rare $\Upsilon(2 S)$ transitions to charmonia and lower bottomonia with the Belle detector
}

\author{
Umberto TAMPONI*† \\ Universita di Torino - INFN, Sezione di Torino. Via P. Giuria 1, 10125 Torino, Italy \\ E-mail: tamponi@to.infn.it
}

\begin{abstract}
In this paper we report the recent results obtained with the $24.7 \mathrm{fb}^{-1}$ sample of $\Upsilon(2 S)$ collected by the Belle experiment. In particular the exclusive search for $\Upsilon(2 S)$ radiative decays to charmonium states is presented. No significant signal was found and new upper limits were set for the branching ratio of these transitions. A new measurement of the branching ratio of the hadronic transition $\Upsilon(2 S) \rightarrow \eta \Upsilon(1 S)$, obtained with the exclusive reconstruction of the $\eta \rightarrow\left(\gamma \gamma, \pi^{+} \pi^{-} \pi^{0}\right)$ and $\Upsilon(1 S) \rightarrow\left(e^{+} e^{-}, \mu^{+} \mu^{-}\right)$final states, is presented.
\end{abstract}

The 2011 Europhysics Conference on High Energy Physics-HEP 2011,

July 21-27, 2011

Grenoble, Rhône-Alpes France

\footnotetext{
* Speaker.

$\dagger$ on behalf of the Belle collaboration
} 


\section{Introduction}

The Belle experiment collected the world largest sample of $\Upsilon(2 S),(158 \pm 4) \times 10^{6} \Upsilon(2 S)$ decays on resonance peak, corresponding to an integrated luminosity of $24.7 \mathrm{fb}^{-1}$. This sample has been exploited during the last year in order to study the transitions from $\Upsilon(2 S)$ to both lower bottomonium and charmonium states. In this paper the results of the search for the radiative decays $\Upsilon(2 S) \rightarrow \gamma_{R} c \bar{c}$ are presented, together with a new measurement of the branching ratio of the hadronic transition $\Upsilon(2 S) \rightarrow \eta \Upsilon(1 S)$.

\section{Charmonium in $\Upsilon(2 S)$ radiative decays}

A systematic search for $\eta_{c}, \chi_{c J}, X(3872), X(3915)$ and $Y(4140)$ signals in $\Upsilon(2 S)$ radiative decays is performed by Belle [1], extending a previous work on the $\Upsilon(1 S)$ transitions to charmonium states [2]. A search for transitions to the hypothetical $X(4350)$, observed as a $3.2 \sigma$ structure in $\gamma \gamma \rightarrow \phi J / \psi[3]$, is performed too. Since any charmonium state above the $\psi^{\prime}$ is predicted to have favoured E1/M1 transitions to $\psi^{\prime}$, the search for new resonances is included looking for the $\gamma_{R} \gamma \psi^{\prime}$ final state where $\gamma_{R}$ is the radiative photon from the $\Upsilon(2 S)$ decay.

$2.1 \Upsilon(2 S) \rightarrow \gamma_{R} X(3872)$

The $X(3872)$ is reconstructed in both the $\pi^{+} \pi^{-} J / \psi$ and $\pi^{+} \pi^{-} \pi^{0} J / \psi$ final state (fig. 1). The $J / \psi$ is detected in the leptonic channels $e^{+} e^{-}$and $\mu^{+} \mu^{-}$, then a kinematic fit with mass constraint is applied to the lepton pair in order to improve the resolution on the $X(3872)$ mass. The radiative photon candidate $\gamma_{R}$ is the highest energy photon in the event with an energy $E^{*}$ in the $e^{+} e^{-}$ center-of-mass frame $(\mathrm{CM})$ greater than $3.5 \mathrm{GeV} / \mathrm{c}^{2}$ and not detected in the forward region of the calorimeter $\left(\cos \left(\theta_{\gamma_{R}}\right)<0.9\right)$. These tecniques for the $\gamma_{R}$ identification and the $\mathrm{J} / \psi$ reconstruction will be applied in every other $\Upsilon(2 S) \rightarrow \gamma_{R} c \bar{c}$ analysis.
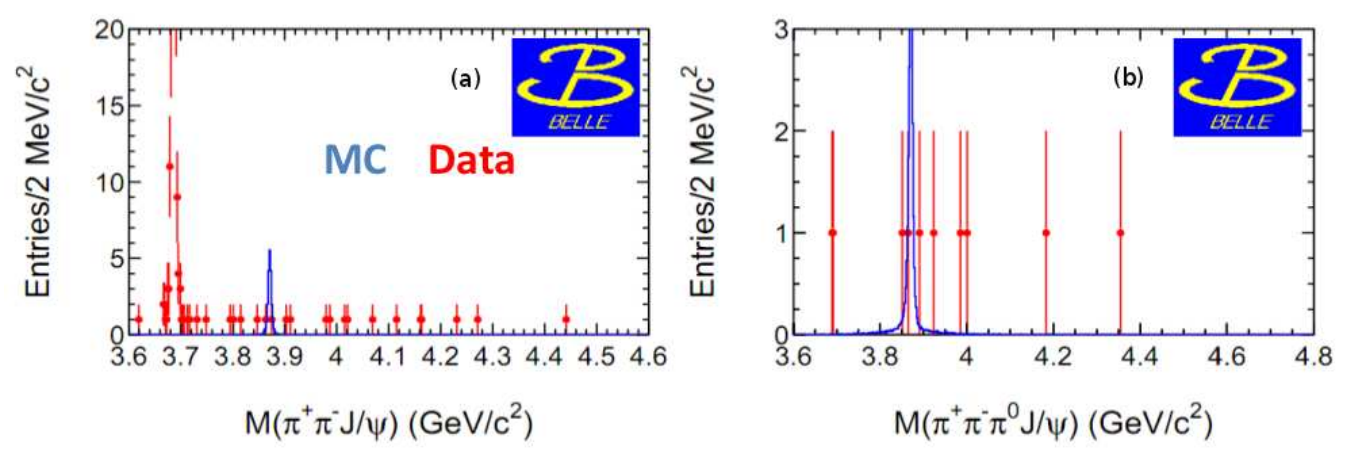

Figure 1: (a) Distribution of the $\pi^{+} \pi^{-} J / \psi$ invariant mass for $\Upsilon(2 S) \rightarrow \gamma_{R} \pi^{+} \pi^{-} J / \psi$ candidates. (b) Distribution of the $\pi^{+} \pi^{-} \pi^{0} J / \psi$ invariant mass for $\Upsilon(2 S) \rightarrow \gamma_{R} \pi^{+} \pi^{-} \pi^{0} J / \psi$ candidates.

No excess of events can be observed in the $X(3872)$ region, and upper the limits at the $90 \%$ C.L. for this transition are set to:

$$
\begin{gathered}
\mathbf{B}(\Upsilon(2 S) \rightarrow \gamma X(3872)) \times \mathbf{B}\left(X(3872) \rightarrow \pi^{+} \pi^{-} J / \psi\right)<0.8 \times 10^{-6} \\
\mathbf{B}(\Upsilon(2 S) \rightarrow \gamma X(3872)) \times \mathbf{B}\left(X(3872) \rightarrow \pi^{+} \pi^{-} \pi^{0} J / \psi\right)<2.4 \times 10^{-6}
\end{gathered}
$$


$2.2 \Upsilon(2 S) \rightarrow \gamma_{R} X(3915)$

The $X(3915)$ is detected in the $\omega J / \psi$ mode, with $\omega \rightarrow \pi^{+} \pi^{-} \pi^{0}$. The selection criteria are the same used in the search for the $X(3872)$ since the final state is almost identical in the two cases. The invariant mass of the $\pi^{+} \pi^{-}$pair is required to be greater than $0.35 \mathrm{GeV} / \mathrm{c}^{2}$, and the $\pi^{0}$ is reconstructed from a pair of photons with invariant mass within $\pm 10 \mathrm{MeV} / \mathrm{c}^{2}$ of the nominal $\pi^{0}$ mass. An upper limit $\mathbf{B}(\Upsilon(2 S) \rightarrow \gamma X(3915)) \times \mathbf{B}(X(3915) \rightarrow \omega J / \psi)<2.8 \times 10^{-6}$ at the $90 \%$ C.L. has been estimated.

$2.3 \Upsilon(2 S) \rightarrow \gamma_{R} X(4350), Y(4140)$

The $X(4350)$ and $Y(4140)$ are both detected in the $\phi J / \psi$ mode, with $\phi \rightarrow K^{+} K^{-}$. The topology of this decay is again very close to the $X(3872) \rightarrow \pi^{+} \pi^{-} J / \psi$ one, thus the selection criteria are very similar. The $\phi$ is reconstructed requiring $1.01 \mathrm{GeV} / \mathrm{c}^{2}<\mathrm{M}\left(\mathrm{K}^{+} \mathrm{K}^{-}\right)<$ $1.03 \mathrm{GeV} / \mathrm{c}^{2}$. The analysis didn't show any excess of events, and the upper limits for these transitions are measured to be $\mathbf{B}(\Upsilon(2 S) \rightarrow \gamma X(4350)) \times \mathbf{B}(X(4350) \rightarrow \phi J / \psi)<1.3 \times 10^{-6}$ and $\mathbf{B}(\Upsilon(2 S) \rightarrow \gamma Y(4140)) \times \mathbf{B}(X(4140) \rightarrow \phi J / \psi)<1.2 \times 10^{-6}$.

$2.4 \Upsilon(2 S) \rightarrow \gamma_{R} \eta_{c}$

The $\eta_{c}$ is reconstructed in five different hadronic final states: $K_{S}^{0} K^{+} \pi^{-}+$c.c., $\pi^{+} \pi^{-} K^{+} K^{-}$, $2\left(K^{+} K^{-}\right), 2\left(\pi^{+} \pi^{-}\right)$and $3\left(\pi^{+} \pi^{-}\right)$. The $K_{S}^{0}$ candidates are reconstructed from $\pi^{+} \pi^{-}$pairs with mass within $\pm 30 \mathrm{MeV} / \mathrm{c}^{2}$ of the $K_{S}^{0}$ nominal mass. The invariant mass distribution of all the reconstructed decay modes is show in fig. 2. The peak due to the $J / \psi$ production via initial state radiation can be clearly seen, but no evidence of $\eta_{c}$ signal has been found. An upper limit of $\mathbf{B}\left(\Upsilon(2 S) \rightarrow \gamma \eta_{c}\right)<2.7 \times 10^{-5}$ can be estimated at the $90 \%$ C.L.

\section{$2.5 \Upsilon(2 S) \rightarrow \gamma_{R} \chi_{C J}$}

The $\chi_{c J}$ candidates are reconstructed with their radiative decays to the $J / \psi$. Thus, together with the high energy photon $\gamma_{R}$ coming from the $\Upsilon(2 S)$ decay, a pair of photon with energy greater than $150 \mathrm{MeV} / \mathrm{c}^{2}$ and a $J / \psi \rightarrow\left(e^{+} e^{-}, \mu^{+} \mu^{-}\right)$candidate are requested in the final state.

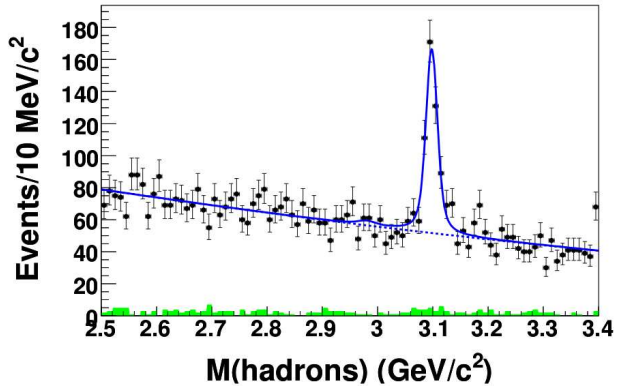

Figure 2: $\eta_{c}$ candidate invariant mass distribu- Figure 3: $\chi_{c J}$ candidate invariant mass distribution in $\Upsilon(2 S) \rightarrow \gamma_{R} \eta_{c}$ events.

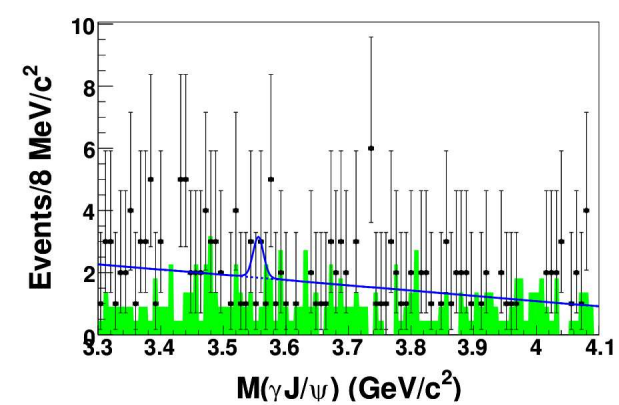

tion in $\Upsilon(2 S) \rightarrow \gamma_{R} \chi_{c J}$ events.

No clear signal can be found (fig. 3), and the upper limits for these transitions have been estimated as: $\mathbf{B}\left(\Upsilon(2 S) \rightarrow \gamma \chi_{c 0}\right)<1.0 \times 10^{-4}, \mathbf{B}\left(\Upsilon(2 S) \rightarrow \gamma \chi_{c 1}\right)<3.6 \times 10^{-6}, \mathbf{B}\left(\Upsilon(2 S) \rightarrow \gamma \chi_{c 2}\right)<$ $1.5 \times 10^{-5}$ at the $90 \%$ C.L. 
$2.6 \Upsilon(2 S) \rightarrow \gamma_{R} X \rightarrow \gamma_{R} \gamma \psi^{\prime}$

In the search for charmonium states decaying into $\gamma \psi^{\prime}$, the $\psi^{\prime}$ is reconstructed via its hadronic decay $\psi^{\prime} \rightarrow \pi^{+} \pi^{-} J / \psi$. Events with four prongs and two photons, one with energy $E^{*}>3.5$ $\mathrm{GeV} / \mathrm{c}^{2}$ and one with $E^{*}>75 \mathrm{MeV} / \mathrm{c}^{2}$ are selected. The primary background, due to the $\psi^{\prime}$ production via initial state radiation, is rejected requiring the squared recoil mass against the $\gamma J / \psi$ candidate to be between $-0.5 \mathrm{GeV}^{2} / \mathrm{c}^{4}$ and $1.5 \mathrm{GeV}^{2} / \mathrm{c}^{4}$.

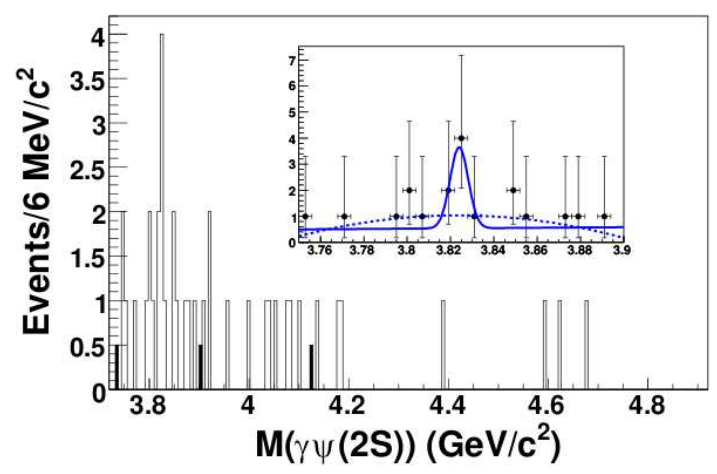

Figure 4: The $\gamma \psi^{\prime}$ invariant mass distribution. The open histogram is from the $\psi^{\prime}$ signal mass region, the shaded histogram is from the normalized $\psi^{\prime}$ mass sidebands. In the inset, the solid curve is the best fit between $3.75 \mathrm{GeV} / \mathrm{c}^{2}$ and $3.90 \mathrm{GeV} / \mathrm{c}^{2}$, and the dashed curve is a fit with only a second-order polynomial to describe the background.

An excess of events with a significance of $1.8 \sigma$ can be seen in the $3.8 \mathrm{GeV} / \mathrm{c}^{2}$ region, as shown in figure 4. The upper limit to this process has been estimated as $\mathbf{B}\left(\Upsilon(2 S) \rightarrow \gamma_{R} X \rightarrow \gamma_{R} \gamma \psi^{\prime}\right)<$ $1.3 \times 10^{-5}$ at the $90 \%$ C.L.

\section{3. $\Upsilon(2 S) \rightarrow \eta \Upsilon(1 S)$}

In the QCDME formalism [4] the $\eta$ transitions between vector quarkonia should be mediated either by two M1 gluons, or by one E1 and one M2 gluon: both cases imply a spin flip of the $b$ quark. The corresponding amplitude should scale as $1 / m_{b}$, and yield information on the chromomagnetic moment of the $b$ quark. By scaling from the $\psi^{\prime} \rightarrow \eta J / \psi$ transition, one expects $\mathbf{B}[\Upsilon(2 S) \rightarrow \eta \Upsilon(1 S)]=8 \times 10^{-4} . \Upsilon(2,3,4 S) \rightarrow \eta \Upsilon(1 S)$ branching ratios have been measured by BaBar [5, 6] and CLEO [7]: they are either unexpectedly large $(\Upsilon(4 S))$, or too small $(\Upsilon(2 S)$ and $\Upsilon(3 S))$.

We search for the $\Upsilon(2 S) \rightarrow \eta \Upsilon(1 S)$ process with the exclusive reconstruction of the $\eta$ in both the $\gamma \gamma$ and the $\pi^{+} \pi^{-} \pi^{0}$ mode. The $\Upsilon(1 S)$ is reconstructed in the leptonic modes $e^{+} e^{-}$ and $\mu^{+} \mu^{-}$. Dominant backgrounds are expected from the transitions $\Upsilon(2 S) \rightarrow \pi \pi \Upsilon(1 S)$ and $\Upsilon(2 S) \rightarrow \gamma \chi_{b J}(1 P) \rightarrow \gamma \gamma \Upsilon(1 S)$, while the radiative Bhabha scattering is the main background processes from the QED continuum.

When the $\Upsilon(1 S)$ is detected in the $e^{+} e^{-}$mode and the $\eta$ in a pair of photons an additional bhabha veto is applied requiring that the electrons are not detected in the forward region of the detector 
$\left(\cos \left(\theta_{e^{-}}^{*}\right)<0.8\right)$. Two mass-constrained fits are applied: the first one in the $\Upsilon(1 S)$ candidate reconstruction, constraining the two lepton candidates to have the $\Upsilon(1 S)$ mass and the second one in order to constrain the $\Upsilon(1 S)$ and the $\eta$ candidates to have the $\Upsilon(2 S)$ invariant mass. A cut on the $\chi^{2}$ of the two fits provides the main background suppression. The mass distribution of the $\eta$ candidate is then fitted with a double gaussian that describes the signal peak, with $\sigma_{\text {peak }}=1.45$ $\mathrm{MeV} / \mathrm{c}^{2}, \sigma_{\text {tail }}=4.54 \mathrm{MeV} / \mathrm{c}^{2}$, mean $\mu=546.99 \mathrm{MeV} / \mathrm{c}^{2}$ and ratio between the two components $\mathscr{R}=\frac{N_{\text {tail }}}{N_{\text {peak }}}=0.09$, and a Crystal ball that describes the background shape (fig. 5). The parameters of the fitting functions are extracted from the MonteCarlo studies, leaving only the signal and background yields as free parameters in the data fit. The number of detected $\Upsilon(2 S) \rightarrow \pi^{+} \pi^{-} \Upsilon(1 S)$ events is used as normalization when calculating the branching ratio in order to avoid the systematic uncertainties on the total number of $\Upsilon(2 S)$ and on the branching ratio of the $\Upsilon(1 S) \rightarrow l^{+} l^{-}$ decays.

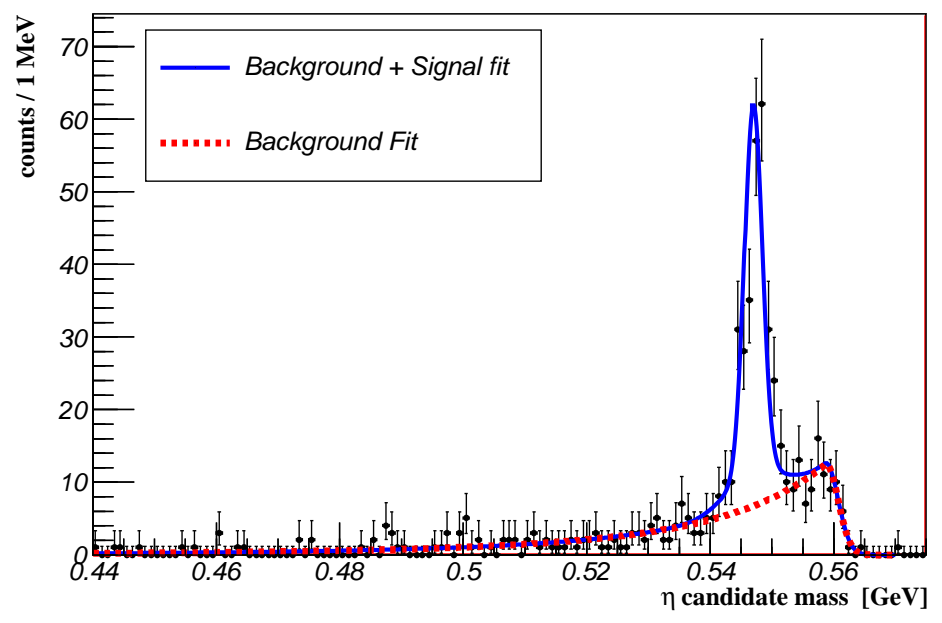

Figure 5: Mass distribution of the $\eta$ in all the four reconstructed final states. $10^{-4}$.

We measure the branching ratio of $\mathbf{B}(\Upsilon(2 S) \rightarrow \eta \Upsilon(1 S))=(3.55 \pm 0.28($ stat $) \pm 0.40($ syst $)) \times$

\section{Conclusions}

We presented the recent results on the $\Upsilon(2 S)$ radiative decays to charmonium states and on the hadronic transitions between lower bottomonia obtained with the Belle detector. No signals of new radiative decays $\Upsilon(2 S) \rightarrow \gamma c \bar{c}$ have been found and an upper limit for the branching ratio of each analyzed transition has been set.

The observation claimed by the CLEO collaboration of the $\Upsilon(2 S) \rightarrow \eta \Upsilon(1 S)$ hadronic transition [7] has been confirmed with high significance, performing the exclusive reconstruction of the $\Upsilon(1 S)$ in its leptonic decays $e^{+} e^{-}$and $\mu^{+} \mu^{-}$. The measured branching ratio $\mathbf{B}(\Upsilon(2 S) \rightarrow \eta \Upsilon(1 S))=$ $(3.55 \pm 0.28$ (stat) $\pm 0.40($ syst $)) \times 10^{-4}$ is higher than the CLEO value [7]. 


\section{References}

[1] X. L. Wang et al. [ Belle Collaboration ], arXiv:1108.4514v1 [hep-ex].

[2] C. P. Shen et al. [ Belle Collaboration ], Phys. Rev. D82, 051504(R) (2010).

[3] C. P. Shen et al. [ Belle Collaboration ], Phys. Rev. Lett. 104, 112004 (2010).

[4] T. -M. Yan, Phys. Rev. D22, 1652 (1980).

[5] B. Aubert et al. [ BaBar Collaboration ], Phys. Rev. D78, 112002 (2008).

[6] J. P. Lees, et al. [ The BaBar Collaboration ], arXiv:1108.5874v1 [hep-ex].

[7] Q. He et al. [ CLEO Collaboration ], Phys. Rev. Lett. 101, 192001 (2008). 